\title{
ELECCIONES LEGISLATIVAS FRANCESAS DE 21 Y 28 DE MARZO DE 1993
}

\author{
CARMEN FERNÁNDEZ-MIRANDA CAMPOAMOR \\ Profesora Titular de Derecho Constitucional \\ Universidad Nacional de Educación a Distancia
}




\section{SUMARIO}

I. Introducción.-II. Campaña electoral.-III. Resultados electoraLES.-IV. VALORACIÓN. 
Revista de Derecho Político, núm. 38, 1994, pp. 361-376

\title{
ELECCIONES LEGISLATIVAS FRANCESAS DE 21 Y 28 DE MARZO DE 1993
}

\author{
POR \\ CARMEN FERNÁNDEZ-MIRANDA CAMPOAMOR \\ Profesora Titular de Derecho Constitucional de la UNED
}

\section{INTRODUCCIÓN}

Los días 21 y 28 de marzo de 1993 se celebran elecciones a la Asamblea Nacional para cubrir 577 escaños, con un mandato de cinco años. El sistema de escrutinio es uninominal mayoritario, a dos vueltas (con excepción de los representantes de ultramar, elegidos en una vuelta). En la primera vence el candidato que haya alcanzado la mayoría absoluta; a la segunda vuelta sólo pueden optar aquellos que hayan logrado un número de votos igual al $12,5 \%$ de los electores de la circunscripción; si no se da este caso, se presentarán los dos candidatos más votados. Serán elegidos quienes logren la mayoría simple.

La trascendencia política de esta consulta se encuentra, en primer lugar, en las previsiones de hundimiento del Partido Socialista, tendencia iniciada en las elecciones regionales del 22 de marzo de 1992 y confirmada por todos los sondeos posteriores. En segundo lugar, y como consecuencia de lo anterior, en el anuncio de una segunda cohabitación entre el Presidente Mitterrand y un Gobierno de centro-derecha emanado de la nueva mayoría en la Asamblea Nacional. 


\section{Cuadro 1}

Siglas de los partidos políticos que compiten en estas elecciones. Líderes principales

\begin{tabular}{|c|c|c|}
\hline Siglas & Partídos políticos & Líderes \\
\hline UPF & Unión Para Francia, coalición que comprende: & \\
\hline RPR & 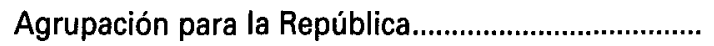 & J. Chirac \\
\hline UDF & Unión para la Democracia Francesa .............................. & V. Giscard d'Estaing \\
\hline PSF & 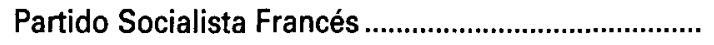 & F. Mitterrand \\
\hline FN & Frente Nacional & J. M. Le Pen \\
\hline \multirow[t]{2}{*}{ PCF } & 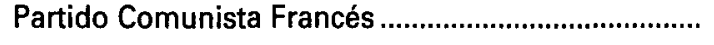 & G. Marchais \\
\hline & Los Ecologistas, coalición que comprende: & \\
\hline Verts & 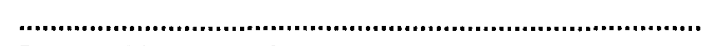 & A. Waetcher \\
\hline GE & 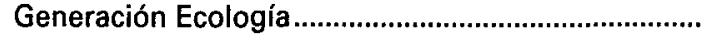 & B. Lalonde \\
\hline MAJ & Mayoría Presidencial & \\
\hline MRG & Movimiento Radical de Izquierda & \\
\hline DVD & Diversos de Derecha & \\
\hline EXD & Extrema Derecha & \\
\hline EXG & Extrema Izquierda & \\
\hline REG & Regionalistas & \\
\hline DIV & Independientes & \\
\hline
\end{tabular}

\section{Cuadro 2}

Resultados de las cuatro últimas elecciones legislativas

\begin{tabular}{lrrrrrrrrr}
\hline & \multicolumn{2}{c}{1978} & \multicolumn{2}{c}{1981} & \multicolumn{2}{c}{1986} & \multicolumn{2}{c}{1988} \\
\cline { 2 - 8 } & $\%$ & Escaños & $\%$ & Escaños & $\%$ & Escaños & $\%$ & Escaños \\
\hline UDF ......... & 22,6 & 154 & 20,8 & 88 & 42,1 & 155 & 19,1 & 130 \\
RPF .......... & 21,4 & 123 & 19,2 & 63 & & 131 & 18,4 & 90 \\
PSF .......... & 22,5 & 115 & 37,5 & 285 & 31,0 & 212 & 34,7 & 275 \\
PCF .......... & 20,5 & 86 & 16,1 & 44 & 9,7 & 35 & 11,3 & 25 \\
FN ............ & & & & & 9,8 & 35 & 9,6 & 1 \\
\hline
\end{tabular}




\section{CAMPAÑA ELECTORAL}

Desde hace un año, los sondeos ponen de manifiesto reiteradamente una alteración de la dinámica política del último decenio, conocido como la "era Mitterrand"; los resultados sobre las intenciones de voto del electorado se repiten una y otra vez, con independencia de la fecha o del medio de comunicación concreto. Por este motivo, la campaña, iniciada el 1 de marzo, se caracteriza por el conocimiento previo de lo que va a suceder, siendo la única duda la gravedad del desastre socialista.

\section{Cuadro 3}

Resumen de la intención de voto del electorado desde enero hasta el 20 de marzo de 1993

(Elaborado con datos de "Le Monde", "Le Nouvel Observateur", "Libération" y "Le Parisien")

\begin{tabular}{|c|c|c|c|}
\hline RPF-UDF & $42 \%$ & $38,5 \%$ & $40 \%$ \\
\hline PS & $22 \%$ & $19,0 \%$ & $18 \%$ \\
\hline Verts-GE & $19 \%$ & $17,0 \%$ & $15 \%$ \\
\hline PC & $8 \%$ & $8,5 \%$ & $9 \%$ \\
\hline FN & $12 \%$ & $11,5 \%$ & $12 \%$ \\
\hline
\end{tabular}

Como puntos destacables del debate preelectoral y de la posición de cada formación política, pueden señalarse los siguientes:

a) Debate público sobre la conveniencia de la dimisión del Presidente Mitterrand, si se confirman las previsiones; se pone en duda la legitimidad de su permanencia al ver cuestionada su política'. El Presidente comunica a la opinión pública su intención de acabar el mandato, con el fin de realizar dos tareas primordiales: la ejecución del Tratado de Maastricht y la reforma de la Constitución ${ }^{2}$.

1 J. DANIEL, editorial de.Le Nouvel Observateur, 3 a 10 de marzo de 1993.

2 El día 9 de noviembre de 1992, el Presidente Mitterrand se dirige a la opinión pública a través de las pantallas de TV comunicando su intención de iniciar el proceso de reforma constitucional. Para ello, y tras informar a los Presidentes de las Cámaras, nombra una Comisión de expertos (2-XII-92) a fin de que elaboren un anteproyecto que recoja su decisión de acortar el mandato presidencial, reforzar los poderes del Parlamento y establecer una legitimación de los ciudadanos para acudir ante el Consejo Constitucional. Días antes de las elecciones la Comisión presenta su trabajo al Jefe del Estado, ante la indiferencia de los partidos y de los medios de comunicación, quienes consideran que ni es el momento adecuado ni el Presidente tiene el respaldo popular necesario para iniciar una reforma. 
Desde la derecha se discute la conveniencia de aceptar la cohabitación o forzar la dimisión del Presidente, manifestándose dos posturas encontradas: mientras Giscard entiende que no se debe aceptar una segunda experiencia política, dado que la primera sólo benefició a Mitterrand, Chirac y Balladur son partidarios de una "cohabitación serena", pero impidiendo el bloqueo presidencial del 86-88 y dejando claro que la Constitución no establece ámbitos reservados al Jefe del Estado, sino ámbitos compartidos por ambas cabezas del Ejecutivo.

b) Días antes de iniciarse la campaña electoral y ante los implacables sondeos, M. Rocard propone la disolución del PS, convirtiéndolo "...en un gran movimiento abierto y moderno, rico en su diversidad, que admita a aquellos que compartan los mismos valores de solidaridad y el mismo objetivo de transformación...n; su oferta se extenderá a ecologistas, reformistas, comunistas, defensores de los derechos humanos y centristas fieles a una tradición social ${ }^{3}$. Es el llamado coloquialmente «bigbang", que pretende dar una esperanza a los simpatizantes socialistas $y$ que, para la derecha, es una búsqueda desesperada de votos por parte del PS, al haber perdido los suyos.

c) Existe el convencimiento de que no es la derecha la que va a ganar, sino el PS a perder; el electorado, más que optar por aquélla, quiere acabar con el Gobierno socialista, con su prepotencia y con su ineficacia. Las razones son múltiples, pudiendo reconducirse a las siguientes.

- Largos años en el poder, con el consiguiente desgaste politico.

- Crisis de identidad y falta de programa político: sus principales temas de enfrentamiento con las fuerzas conservadoras ya han sido asumidos por éstas, que, a su vez, han marcado una conveniente distancia con la extrema derecha.

- División interna del partido como consecuencia de la lucha por suceder a Mitterrand.

- Continuas acusaciones de corrupción.

3 Ver Le Monde, Libération, Le Figaro..., del día 19 de febrero de 1993. Igualmente, ver en Cambio16 un extracto del discurso de M. Rocard en "Socialismo. El fin de un sueño", 17 de mayo de 1993. 
- Por último, y lo más importante, grave crisis económica, con un aumento continuo del paro, cifrado durante la campaña en tres millones de personas sin trabajo ${ }^{4}$.

d) El RPR y la UDF, formando la coalición "Unión para Francia", presentan candidaturas conjuntas en 497 de las 577 circunscripciones. Ambos partidos pactan que será Primer Ministro un miembro de la formación que obtenga más votos. La derecha recoge el cansancio socialista y es consciente de que va a obtener una victoria envenenada, por las siguientes razones:

- Va a heredar una difícil situación económica, que habrá de gestionar en un tiempo muy corto y transitorio, hasta las elecciones presidenciales de 1995: va a administrar la crisis.

- Pese a su única voz de cara al electorado, la coalición tiene problemas internos, tanto respecto a la política europea ${ }^{5} \mathrm{co}$ mo al intento de los líderes de ambas formaciones - Chirac y Giscard- de suceder a Mitterrand en la Presidencia de la República.

e) El Frente Nacional mantiene su programa habitual y sus excesos verbales; sin embargo, la sensación general es que ha alcanzado su techo electoral. Las circunstancias especiales de esta campaña le sitúan en una posición muy poco relevante, a diferencia de otras convocatorias: la derecha no necesita su apoyo y la izquierda está demasiado ocupada en intentar frenar la sangría de votos que se anuncia.

f) Los Ecologistas — Los Verdes y Generación Ecología- presentan candidaturas conjuntas ${ }^{6}$ para lograr afirmarse como partido parlamentario. Los sondeos le vaticinan un apoyo importante del electorado, lo que lleva al PS a ofrecerles su inclusión en el proyecto de Rocard e, incluso, a apoyarles en la segunda vuelta electoral, propuestas no aceptadas por aquéllos.

4 J. Daniel, "Amenazas electorales en Francia", en El País, 21-III-93. El paro es el eje de toda la campaña; el francés que no está en paro - dice este comentarista- piensa que puede estarlo y está seguro de que sus hijos lo sufrirán.

5 No hay que olvidar que en el referéndum sobre el Tratado de Maastricht, la coalición mantuvo diferentes posturas: mientras que la formación de Giscard se manifestó a favor, en el RPR, si bien Chirac defendió el voto afirmativo, Ch.Pasqua y P. Seguin hicieron campaña por el no.

${ }_{6}$ En las elecciones regionales de 1993 obtuvieron, por separado, un 7,1\% GE y un $6,8 \%$ los Verdes, es decir, casi un $14 \%$, lo que propiciará el coaligarse posteriormente. 
g) Finalmente, el PC, con un techo electoral claro, parece que mantiene un sector de incondicionales que apoyan su programa, pese a los cambios sufridos por estos movimientos políticos en toda Europa; también a ellos se dirige la oferta de renovación de la izquierda lanzada por Rocard.

\section{RESULTADOS ELECTORALES}

1. Primera vuelta (21 de marzo de 1993)

\section{Cuadro $4^{7}$}

Resultados según el Ministerio del Interior (A excepción de Guayana, Guadalupe y Martinica)

\begin{tabular}{|c|c|c|}
\hline Partidos & Votos & $\%$ \\
\hline EXG & 448.784 & 1,77 \\
\hline PCF & 2.323 .437 & 9,21 \\
\hline PS & 4.444.761 & 17,62 \\
\hline MRG & 228.758 & 0,90 \\
\hline MAG & 390.401 & 1,54 \\
\hline GE & 921.925 & 3,65 \\
\hline Verts & 1.022 .663 & 4,05 \\
\hline REG & 104.972 & 0,41 \\
\hline DIV & 957.711 & 3,79 \\
\hline RPR & 5.133 .870 & 20,35 \\
\hline UDF & 4.849.489 & 19,22 \\
\hline DVD & 1.164.377 & 4,61 \\
\hline FN & 3.158 .843 & 12,52 \\
\hline EXD & 69.985 & 0,27 \\
\hline
\end{tabular}

, Fuente: Embajada de Francia. 
Cuadro $5^{8}$

\begin{tabular}{|c|c|c|}
\hline \multicolumn{3}{|c|}{ Resumen de los resultados de la 1." vuelta } \\
\hline & Partidos & $\%$ \\
\hline 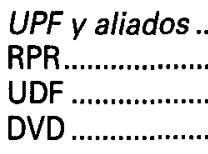 & (1) & $\begin{array}{r}44,18 \\
20,35 \\
19,22 \\
4,61\end{array}$ \\
\hline 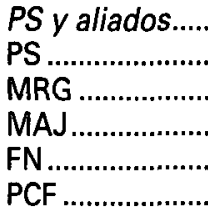 & (1) & $\begin{array}{r}20,06 \\
17,62 \\
0,90 \\
1,54 \\
12,52 \\
9,21\end{array}$ \\
\hline 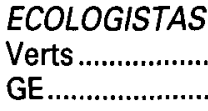 & (1) & $\begin{array}{l}7,70 \\
4,05 \\
3,65\end{array}$ \\
\hline 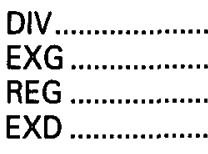 & (1) & $\begin{array}{l}3,79 \\
1,77 \\
0,41 \\
0,27\end{array}$ \\
\hline
\end{tabular}

\section{Cuadro $6^{9}$}

\section{Las doce grandes batallas electorales}

\begin{tabular}{|c|c|c|}
\hline & Localidad - Departamento & Candidatos enfrentados \\
\hline & CONFLANS - Yvelines & M. Rocard (PS) - P. Cardo (RPR) \\
\hline 2. & SARLAT - Dordogne ................. & R. Dumas (PS) - J. de Peretti (RPR) \\
\hline 3. & VILLEJUIF - Val de Marne ...................... & G. Marchais (PC) - D. Richard (RPR) \\
\hline 4. & DOLE - Jura .......................... & D. Voinet (Verdes) - G. Barbier (UDF) \\
\hline 5. & ELBEUF - Seine Maritime... & L. Fabius (PS) - M. Baldeneeck (UDF) \\
\hline 6. & DREUX - Eure et Loire ....... & F. Stibois (FN) - G. Hamel (RPR) \\
\hline & NEVERS - Nievre .......... & P. Bérégovoy (PS) - D. Rostein (RPR) \\
\hline 8. & BLOIS - Loire et Cher...... & J. Lang (PS) - J. Gourault (UDF) \\
\hline 9. & SARCELLES - Val D'Oise .. & P. Lellouche (RPR) - D. Strauss-Kahn (PS) \\
\hline 10. & NIZA - Alpes Maritimes ........................... & J. M. Le Pen (FN) - R.Salles (UDF) \\
\hline 11. & GARDANNE - Bouches du Rhone..... & B. Tapie (PS) - H. Fabre-Aubespry (RPR) \\
\hline 12. & 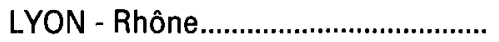 & M. Noir (ex RPR) - A. Mérieux (RPR) \\
\hline
\end{tabular}

\& El País, 23-III-93.

9 El Mundo, 28-III-93. 
Los resultados de la primera vuelta pueden resumirse con las siguientes palabras: «...Victoria sin paliativos del centro-derecha, un retroceso impresionante del PS, una inesperada caída de los Ecologistas, un porcentaje significativo de abstención y un ligero repunte del FN. EI PC mantiene el mismo número de escaños" ${ }^{10}$.

La prensa francesa del día 22 califica de "maremoto" el castigo recibido en las urnas por el PS, del que asombra, no su realización, sino sus dimensiones ". Sobre ello se mantienen dos criterios:

- Mientras que un sector de opinión atribuye el desastre electoral al paro, los escándalos y el desgaste del poder ${ }^{12}$,

- Otro sector entiende que tal derrota responde a razones que trascienden a la situación política concreta: la consumación de las ofertas clásicas del socialismo y el fracaso de lo sistemas comunistas, con la consiguiente crisis de la izquierda ${ }^{13}$.

M. Rocard hace un llamamiento a la izquierda, que no logró ningún escaño en la primera vuelta, para que acuda a las urnas y respalde a los candidatos con más posibilidades, a fin de paliar la hegemonía del centro-derecha en la Asamblea ${ }^{14}$.

El adverso resultado de los Ecologistas ha sido la segunda sorpresa del escrutinio frente a las esperanzas despertadas por los sondeos de todo un año; el electorado no ha dado su confianza a un programa poco pragmático, en una situación de crisis necesitada, esencialmente, de buenos gestores públicos. Pese a ello, la coalición mantiene su negativa de pacto con un PS totalmente desprestigiado, pese a las ofertas, interesadas, de aquél.

10 G. Santesmases, "Aprender de lo ocurrido" en El Mundo, 22-III-93.

11 J. RuPÉREZ, "La rosa ha perdido su nombre", en El Mundo, 22-III-93.

12 El secretario general del PS, L. Fabius, manifiesta que estos errores políticos han generado los resultados adversos. Ver editorial de Diario 16 y El País de 22-III-93.

P. GonZÁlez, «Empieza el post-socialismo», Diario 16, 22-III-93.

M. RocARD, "Francia necesita una izquierda", en El Mundo, 28-III-93. 


\title{
2. Segunda vuelta (28 de marzo de 1993)
}

\author{
Cuadro $7^{15}$
}

Resultados de la segunda vuelta según el Ministerio del Interior (sobre 497 circunscripciones)

(1.: 33.773.804; V.: 22.802.301; E.: 20.632.930; Abs.: 32,48\%)

\begin{tabular}{|c|c|c|}
\hline Partidos & Votos & $\%$ \\
\hline EXG & 21.509 & 0,10 \\
\hline PCF & 951.213 & 4,61 \\
\hline PS & 5.829 .493 & 28,25 \\
\hline MRG & 237.622 & 1,15 \\
\hline MAJ & 448.187 & 2,17 \\
\hline GE & 17.403 & 0,08 \\
\hline 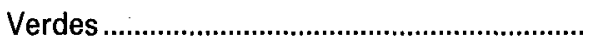 & 20.088 & 0,09 \\
\hline REG & 36.971 & 0,17 \\
\hline DIV & - & - \\
\hline RPR & 5.832 .987 & 28,27 \\
\hline UDF & 5.331 .935 & 25,84 \\
\hline DVD & 736.372 & 3,56 \\
\hline FN & 1.168 .150 & 5,66 \\
\hline EXD & - & - \\
\hline
\end{tabular}

Cuadro $8^{16}$

Resultado de los grandes duelos electorales

\begin{tabular}{|c|c|c|}
\hline & Localidad - Departamento & Candidato vencedor - Candidato derrotado \\
\hline & CONFLANS - Yvelines & P. Cardo (UDF) - M. Rocard (PS) \\
\hline 2. & SARLAT - Dordogne ............ & J. J. de Peretti (RPR) - R. Dumas (PS) \\
\hline 3. & VILLEJUIF - Val de Marne ..................... & G. Marchais (PC) - D. Richard (RPR) \\
\hline 4. & 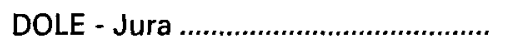 & G. Barbier (UDF) - D. Voynet (Verdes) \\
\hline 5. & ELBEUF - Seine Maritime. & L. Fabius (PS) - M. Baldeneeck (UDF) \\
\hline 6. & DREUX - Eure et Loire .............................. & G. Hamel (RPR) - M. F. Stirbois (FN) \\
\hline & NEVERS - Nievre ......... & P. Bérégovoy (PS) - D. Rostein (RPR) \\
\hline 8. & BLOIS - Loire et Cher ....... & J. Lang (PS) - J. Gourault (UDF) \\
\hline 9. & SARCELLES - Val D'Oise .... & P. Lellouche (RPR) - D. Strauss-Kahn (PS) \\
\hline 10. & & JR. Salles (UDF) - J. M. Le Pen (FN) \\
\hline 11. & GARDANNE - Bouches du Rhone..... & B. Tapie (PS) - H. Fabre-Aubespry (RPR) \\
\hline 12. & LYON - Rhône & M. Noir (ex RPR) - A. Mérieux (RPR) \\
\hline
\end{tabular}

15 Fuente: Embajada de Francia.

16 El Pais, 30-III-93. 


\section{Cuadro $9^{17}$}

Resumen de los escaños, con la variación experimentada respecto a la Asamblea Nacional anterior

\begin{tabular}{|c|c|c|c|c|}
\hline Partidos & $1 .^{D}$ vuelta & $2 . \stackrel{s}{ }$ vuelta & Total & Diferencia \\
\hline UPF y aliados ............. & 80 & 404 & 484 & \\
\hline RPF & 42 & 205 & 247 & +121 \\
\hline UDF $\ldots \ldots \ldots \ldots \ldots$ & 36 & 177 & 213 & +82 \\
\hline DVD ........................... & 2 & 22 & 24 & +13 \\
\hline PS y aliados................. & 0 & 70 & 70 & \\
\hline PS & 0 & 54 & 54 & -198 \\
\hline MRG $\ldots . . . \ldots \ldots \ldots \ldots \ldots$ & 0 & 6 & 6 & -4 \\
\hline MAJ............................ & 0 & 10 & 10 & -10 \\
\hline PCF & 0 & 23 & 23 & - \\
\hline
\end{tabular}

En las 497 circunscripciones en "ballotage" se confirman los resultados de la primera vuelta ${ }^{18}$; numerosas personalidades del PS y del mismo Gobierno son derrotadas por los adversarios del centro-derecha, entre ellos el propio candidato presidencial socialista, M. Rocard.

EI PC logra los votos suficientes para poder formar grupo parlamentario; por el contrario, y por efecto del sistema mayoritario, el Frente Nacional no llega a la Asamblea, pese a tener más votos que aquéllos. La coalición Ecologista queda muy lejos de la Cámara.

El centro-derecha ratifica su victoria de forma tan contundente, que impedirá cualquier oposición a su acción de gobierno; el RPR logra más votos y escaños que su socio en la coalición, por lo que a esa formación corresponde la designación del Primer Ministro, como se había pactado; Balladur será el candidato que reúna las cualidades de eficacia, moderación y europeísmo esenciales para afrontar la nueva cohabitación con el Presidente de la República. Como dice el comentarista político DUHAMEL ${ }^{19}$, si bien en la época de De Gaulle, Pompidou y Giscard la derecha controlaba la Jefatura del Estado y la Asamblea Nacional, tenía enfrente una oposición combativa, tanto en el terreno sindical como muni-

17 El País, 30-III-93.

is P. GonzÁlez, «El fin de un ciclo histórico»; J. M. THENARD, «El relevo socialista", en El Mundo, 29-III-93.

ig El País, 22-1II-93. 
cipal; en este momento sólo tienen enfrente al enfermo y desprestigiado Presidente Mitterrand. Efectivamente, el centro-derecha domina el Parlamento, el Gobierno, todos los Consejos Regionales, salvo tres ${ }^{20}$, y un número importante de alcaldías ${ }^{21}$.

De los resultados parece deducirse, en primer lugar, una importante crisis política y moral, que se manifiesta en el rechazo a los modos de gobierno del último decenio; en segundo lugar, una gran preocupación por la alta cifra de paro, y, en consecuencia, la búsqueda de gestores más prudentes o reticentes respecto a la aplicación del Tratado de Maastricht ${ }^{22}$.

\section{La abstención y los votos en blanco}

Abstención normal en una situación política difícil, que corresponde esencialmente al electorado de izquierdas.

1.․․ vuelta

Este ligero aumento en la segunda votación puede explicarse por varias razones. En primer lugar, porque la mayoría de los enfrentamientos electorales de la segunda vuelta son entre la derecha y la extrema derecha -81-, no afectando al electorado de izquierdas, que o bien se abstiene o vota en blanco; en segundo lugar porque, dado el hundimiento del PS en la primera vuelta, parte de sus votantes entiende que nada va a cambiar el veredicto de las urnas, lo que no se corresponde con lo sucedido realmente ${ }^{23}$.

20 Dos presididos por el PS (Limousin y Borgoña) y uno por Los Verdes (Norte-Paso de Calais).

${ }^{21}$ Entre las alcaldías de ciudades de más de 300.000 habitantes, 70 son del PS, 48 del PC y 84 del centro-derecha.

${ }_{22}$ La prensa se hace eco del ataque a la "política europea demasiado confiada" por parte de las zonas más deprimidas del país. Ver editorial de Diario 16, $22-$ III-93.

${ }^{23}$ Como ejemplo, en los duelos electorales más llamativos (cuadro 7.ํ), el PS compitió en 7 y ganó en 4; en el total, si en la primera vuelta los candidatos lograron sólo el $18 \%$, en la segunda alcanzaron el $30 \%$. Ver sobre esto $A$. Chaussebourg, "La droite enlève plus de 480 sièges, la gauche 90 ", Le Monde, 29III-93. 
Por último, hay que señalar el elevado número de votos en blanco, que manifiestan un criterio que, si bien respalda el sistema democrático ${ }^{24}$, rechaza tanto los partidos concretos como la dinámica política de la V República.

1. vuelta

1.417 .774 votos

2." vuelta

2.169.371 votos

\section{VALORACIÓN}

La Constitución de 1958 establece un sistema parlamentario dualista, en el que el Gobierno está sometido a una doble confianza: del Jefe del Estado y de la Asamblea Nacional, ambos elegidos por sufragio universal directo; sin embargo, los factores políticos concretos afectan directamente al esquema jurídico constitucional. La existencia habitual de mayorías afines ha dado una preeminencia clara al Presidente de la República, situándole en la posición de líder de la mayoría unívoca: controla el Parlamento y asume la dirección política, convirtiendo al Primer Ministro en instrumento dócil de su política. Esto fue lo ocurrido desde el inicio de la V República hasta 1986, con los Presidentes De Gaulle, Pompidou, Giscard y Mitterrand.

En la fecha señalada, y con motivo de las elecciones legislativas de 16 de marzo, se modificaron los factores políticos, generando una mayoría parlamentaria contrapuesta a la presidencial y, en consecuencia, un equilibrio de poderes distinto: el líder de la mayoría parlamentaria dirige el Gobierno, no necesitando más confianza que la de la Cámara; el Presidente de la República se repliega hacia la posición clásica de Jefe de Estado parlamentario, representante de la nación y garante de su unidad y permanencia. Durante la llamada cohabitación política, de 1986 a 1988, se demostró cómo un Primer Ministro con una mayoría parlamentaria favorable, posee los medios legales adecuados para convertirse en el centro de impulsión política e imponerse al Presidente de la República, en contra de la práctica iniciada por el General De Gaulle y seguida por sus sucesores $^{25}$.

${ }^{24}$ El voto antisistema se encuentra esencialmente en el sector partidario de Le Pen; no hay que olvidar que, pese a no haber logrado ningún escaño, su número de votantes ha aumentado ligeramente.

${ }_{25}$ Es preciso señalar que posteriormente, en las elecciones legislativas de 1988, tras la reelección de Mitterrand, la Asamblea Nacional recibe una representa 
Las elecciones legislativas de 21 y 28 de marzo de 1993 han supuesto un cambio político espectacular, que inicia una segunda cohabitación con acentos propios, debido a la hegemonía del centro-derecha en la Asamblea Nacional, a la ausencia de una oposición contundente $y$ al desprestigio y fin de mandato del Presidente Mitterrand. Pese a las sucesivas declaraciones del Jefe del Estado reivindicando un dominio "reservado", éste no existe jurídicamente; a ello hay que añadir la desvalorización de los poderes constitucionales propios del Presidente, en este contexto político. Veámoslo, de forma resumida, ya que no es éste el lugar adecuado para hacer un estudio completo.

Los poderes "reservados» del Presidente de la República, defensa y exteriores, no provienen de la Constitución, sino de la práctica política generada por la larga coincidencia de mayorías, unido a la ambigüedad de la Norma ${ }^{26}$. Las competencias son legalmente compartidas por ambos titulares del Ejecutivo, lo que, en la actual coyuntura política, da paso a un acuerdo necesario, ya que del Gobierno depende la ejecución de las decisiones y de la Cámara la votación de los créditos, la aprobación de las Leyes o la autorización de los Tratados; ambos poderes no son del Presidente Mitterrand, sino del Primer Ministro de centro-derecha.

Respecto a los poderes constitucionales propios no necesitados de refrendo, en concreto el nombramiento y revocación del Primer Ministro, la convocatoria de referéndum, la declaración de dictadura constitucional y la disolución de la Asamblea Nacional, las cautelas legales establecidas por la Norma, inclinan la balanza, en estas circunstancias políticas concretas, del lado del Gobierno. En el nombramiento y cese del Primer Ministro, el Presidente de la República está totalmente condicionado por la confianza de la Cámara. Para convocar un referéndum precisa la propuesta del Gobierno o de las Cámaras, por la que sobra todo comentario. La disolución de la Asamblea exige la previa consulta, no vinculante, al Primer ministro y a las Presidentes de las Cámaras; sin embargo, la legi-

ción del PS de 275 Diputados, 220 del centro-derecha, 25 del PC y 1 del FN; ningún partido, pues, se acerca a la mayoría absoluta, con lo que es el único momento en la V República en que se lleva a la práctica el sistema de doble confianza. Mitterrand nombra Primer Ministro a $M$. Rocard, persona de su partido pero con el que no mantiene buenas relaciones; es, sin embargo, capaz de pactar con la Asamblea Nacional (ver cuadro 2.\%).

${ }_{26}$ El artículo 15 de la Constitución establece que el Presidente de la República es el Jefe de los Ejércitos y preside los Consejos y Comités Superiores de la Defensa Nacional. Sin embargo, en el artículo 20 atribuye al Gobierno el determinar y conducir la política de la nación y disponer de la Administración y de las FAS. A su vez, el artículo 21 dice que el Primer Ministro dirige la acción de gobierno y es responsable de la Defensa Nacional. 
timidad recién revalidada de la mayoría convierte su disolución, en contra de las opiniones recabadas, en causa de grave inestabilidad política y en actuación muy peligrosa para el Jefe del Estado. Finalmente, para adoptar las medidas excepcionales previstas en el artículo 16 de la Constitución ha de consultar al Primer Ministro, a los Presidentes de las Cámaras y al Consejo Constitucional; la declaración de tal estado en contra del criterio de, al menos, los tres primeros, sería tan arriesgado políticamente que parece inviable su utilización.

Además de lo dicho, la situación concreta del Presidente Mitterrand es, políticamente, mucho más débil que la correspondiente a la etapa $1986-88$.

- En la primera cohabitación, Mitterrand tenía un importante prestigio político, un partido cohesionado y un grupo parlamentario con 212 Diputados que respaldaban sus continuos bloqueos a la política realizada, efectivamente, por el Primer Ministro Chirac. En la actualidad, el Presidente Mitterrand está desprestigiado políticamente, con un partido hundido y dividido y un grupo parlamentario formado por 70 Diputados.

- En 1986 Mitterrand tenía la posibilidad de ser reelegido para un segundo mandato y la necesidad de demostrar su papel moderador en una difícil situación política, reconocido por el electorado en 1988 con su reelección. En 1993, el Presidente finaliza su carrera política y sólo le retiene en el pode su propia ambición personal en la tarea europea y la, quizás, sentida obligación de dar un margen de tiempo al PS para recomponerse de cara a las presidenciales de 1995. Sólo eso explica su empeño en no dimitir, pese a los contundentes resultados electorales.

Como conclusión desde los resultados electorales analizados, el Gobierno, apoyado por una mayoría parlamentaria muy abultada, será el centro de impulsión política en toda esta etapa; el Presidente de la República pasa a ocupar la posición de un Jefe de Estado parlamentario clásico, representante de la nación. La política que se lleve a cabo será la del Primer Ministro y en ningún sentido la del Presidente Mitterrand; si éste tiene algún protagonismo es porque al Gobierno le conviene.

Madrid, mayo 1993 